\title{
Corrigendum to "Probiotics, prebiotics, and synbiotics regulate the intestinal microbiota differentially and restore the relative abundance of specific gut microorganisms" (J. Dairy Sci. 103:5816-5829)
}

\section{Chen Li, Zhihua Niu, Meijuan Zou, Suyue Liu, Miaoshu Wang, Xinxi Gu, Haiqiang Lu, Hongtao Tian, and Rajesh Jha}

Page 5825 shows an incorrect Figure 5. The correct version of Figure 5 is shown below.

The authors regret the error.

\section{REFERENCES}

Li, C., Z. Niu, M. Zou, S. Liu, M. Wang, X. Gu, H. Lu, H. Tian, and R. Jha. 2020. Probiotics, prebiotics, and synbiotics regulate the intestinal microbiota differentially and restore the relative abundance of specific gut microorganisms. J. Dairy Sci. 103(7):5816-5829. https://doi.org/ $10.3168 /$ jds.2019-18003.
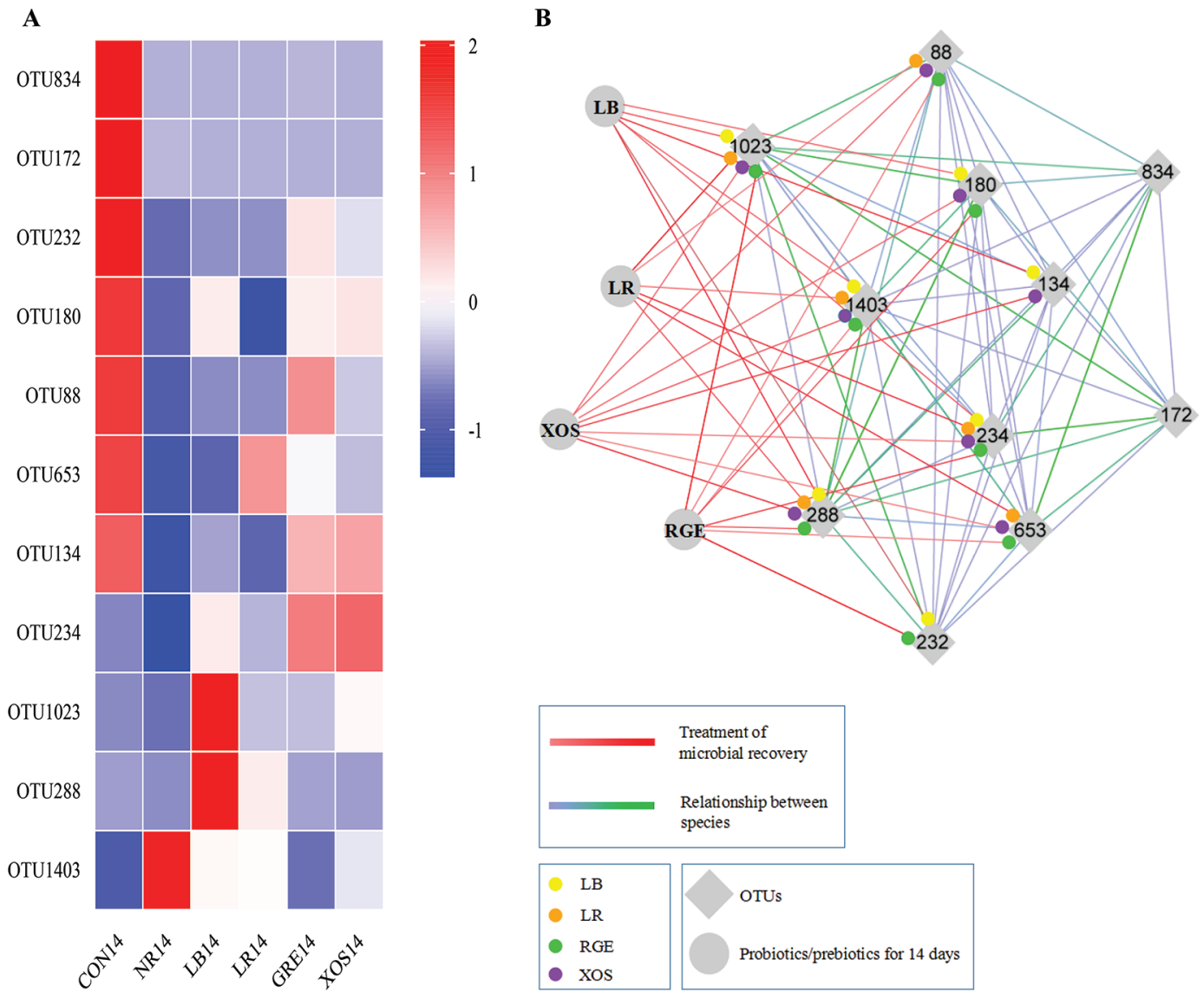

Figure 5. Effects of different treatments on intestinal microbes that cannot be naturally recovered in a short time. (A) Heat map displaying the relative abundance of operational taxonomic units (OTU) of the 11 microorganisms that cannot be naturally recovered in a short time in the 6 groups (CON14, NR14, LB14, LR14, RGE14, XOS14, where 14 indicates treatment for $14 \mathrm{~d}$ ). Relative abundance is based on the number of high-throughput sequencing reads clustering into each OTU after normalizing the number of reads per sample. Each column in the heat map represents one sample, and each row represents one genus. The relative abundance of each genus is indicated by a gradient of color from blue (low abundance) to red (high abundance). (B) Correlation network: specific OTU reversed by the different treatments are significantly correlated. Circle, the treatment of the supplement for $14 \mathrm{~d}$; diamond, OTU; red line, different treatment recovery OTU; blue line, negative correlation between OTU; green line, positive correlation between OTU. CON = control; $\mathrm{NR}=$ natural recovery group; LB = Lactobacillus bulgaricus treatment for $14 \mathrm{~d}$; LR = Lactobacillus rhamnosus treatment for $14 \mathrm{~d}$; XOS = xylooligosaccharide treatment for $14 \mathrm{~d}$; RGE $=$ red ginseng hydrolysate treatment for $14 \mathrm{~d}$. 